International Journal of Advanced Astronomy, 5(1)(2017) 1-6
International Journal of Advanced Astronomy
SPC
Website: www.sciencepubco.com/index.php/IJAA
doi:
Research paper, Short communication, Review, Technical paper

\title{
A study of libration points in CR3BP under albedo effect
}

\author{
M. Javed Idrisi* \\ Department of Mathematics, Al-Falah University, Faridabad (Haryana) - 121004, India \\ *Corresponding author E-mail:mjavedidrisi@gmail.com
}

\begin{abstract}
In this paper this is investigated how albedo perturbed the libration points from its original position? It is found that there exist five libration points, three collinear and two non-collinear and all the libration points are affected by Albedo. The non-collinear libration points are stable for a critical value of mass parameter $\mu \leq \mu_{c}$, where $\mu_{\mathrm{c}}=\mu_{o}-(0.00891747+0.222579 k) \alpha\left(\mu_{o}\right.$ is the critical mass parameter for classical case) but collinear libration points are still unstable.
\end{abstract}

Keywords:Restricted Three-Body Problem; Radiation Pressure; Albedo Effect; Libration Points; Stability.

\section{Introduction}

The restricted three-body problem is one of well known problem in the field of celestial mechanics in which two finite bodies called primaries move around their center of mass in circular or elliptic orbits under the influence of their mutual gravitational attraction and a third body of infinitesimal mass is moving in the plane of the primaries which is attracted by the primaries and influenced by their motion but not influencing them. In classical case there exist five libration points out of which three are collinear and two are non-collinear. The collinear libration points $L_{1}, L_{2}$ and $L_{3}$ are unstable for $0 \leq \mu \leq 1 / 2$ and the non collinear libration points $L_{4,5}$ are stable for a critical value of mass parameter $\mu<\mu_{\mathrm{c}}=0.03852 \ldots$, Szehebely [2]. Some studies related to the equilibrium points in $\mathrm{R} 3 \mathrm{BP}$ or ER3BP, taken into account the oblateness and triaxiality of the primaries, Coriolis and Centrifugal forces, Yarkovsky effect, variation of the masses of the primaries and the infinitesimal mass etc. are discussed by Danby [1]; Vidyakin [5]; Sharma [6]; Subbarao and Sharma [7]; Choudhary R. K. [9]; Cid R. et. al. [14]; ElShaboury [19]; Bhatnagar et al. [22]; Selaru D. et.al. [24]; Markellos et al. [25]; Subbarao and Sharma [26]; Khanna and Bhatnagar [27, 29]; Roberts G.E. [33]; Oberti and Vienne [34]; Perdiou et. al. [36]; Sosnytskyi [38]; Ershkov [46]; Arredondo et.al. [47]; Idrisi and Taqvi [48]; Idrisi [49]; Idrisi and Shalini [59]; Idrisi and Jain [60].

The photo-gravitational restricted three-body problem arises from the classical problem if one or both primaries is an intense emitter of radiation, formulated by Radzievskii (1950). He has considered only the central forces of gravitation and radiation pressure on the particle of infinitesimal mass without considering the other two components of light pressure field and studied this problem for three specific bodies; the Sun, a planet and a dust particle. The radiation repulsive force $F_{p}$ exerted on a particle can be represented in terms of gravitational attraction $F_{\mathrm{g}}$ (Radzievskii, $1950)$ as $F_{p}=F_{g}(1-q)$, where $q=1-F_{p} / F_{\mathrm{g}}$, a constant for a given particle, is a reduction factor expressed in terms of the particle radius $a$, density $\delta$ and radiation-pressure efficiency factor $x$ (in c.g.s. system) as: $q=1-\frac{5.6 \times 10^{-3}}{a \delta} x$

The assumption that ' $q$ ' is a constant implies that the fluctuations in the beam of solar radiation and the effect of planet's shadow are neglected. Typical values for diameter of IDP (Interplanetary Dust Particles) are in the range of $50-500 \mu \mathrm{m}$ and their densities range is $1-3 \mathrm{~g} / \mathrm{cm}^{3}$ with an average density of $2 \mathrm{~g} / \mathrm{cm}^{3}$. As the size of the particles increases, their density decreases (Grün et.al. [32]). Some of the notable research in PRTBP are carried by Chernikov [4]; Bhatnagar and Chawla [11]; Schuerman D.W [12]; Kunitsyn and Tureshbaev [15]; Simmons et. al. [16]; Sharma [17]; Lukyanov [18]; Xuetang et.al. [21]; Ammar [40]; Douskos [42]; Singh and Leke [43]; Katour et.al. [50] etc. In 2012, S. V. Ershkov [46] studied the Yarkovsky effect in generalized photogravitational 3body problem and proved the existence of maximally 256 different non-planar equilibrium points when second primary is nonoblate spheroid. The main contribution of the natural radiation pressure on the satellite is due to the direct solar radiation and the second main contribution of radiation forces is due to the Earth reflected radiation known as the Albedo studied by Anselmo et.al. [13]; Nuss [28]; McInnes [31]; Bhanderi [35]; Pontus [37]; MacDonald [45] etc. Albedo effect is one of the most interesting nongravitational force having significant effects on the motion of infinitesimal mass. Albedo is the fraction of solar energy reflected diffusely from the planet back into space (Harris and Lyle [3]). It is the measure of the reflectivity of the planet's surface. Therefore, the Albedo can be defined as the fraction of incident solar radiation returned to the space from the surface of the planet (Rocco [41]) as

$$
\text { Albedo }=\frac{\text { radiation reflected back to the space }}{\text { incident radiation }} \text {. }
$$

In this paper the Albedo effect on the existence and stability of the libration points when smaller primary is a homogeneous ellipsoid has been studied. This paper is divided into five sections. In section-2, the equations of motion are derived. The existence of noncollinear and collinear libration points is shown in section-3. In section-4, the stability of non-collinear and collinear libration points is discussed. In the last section, all the results are discussed. 


\section{Equations of motion}

Let $m_{i}(i=1,2)$ be point masses such that $m_{1}>m_{2}$ and $m_{1}$ is a source of radiation, are moving in the circular orbits around their center of mass $O$. An infinitesimal mass $m_{3} \ll 1$, is moving in the plane of motion of $m_{1}$ and $m_{2}$. The distances of $m_{3}$ from $m_{1}, m_{2}$ and $O$ are $\boldsymbol{r}_{1}, \boldsymbol{r}_{\mathbf{2}}$ and $\boldsymbol{r}$ respectively. $\boldsymbol{F}_{\mathbf{1}}$ and $\boldsymbol{F}_{\mathbf{2}}$ are the gravitational forces acting on $m_{3}$ due to $m_{1}$ and $m_{2}$ respectively, $\boldsymbol{F}_{\boldsymbol{p}}$ is the solar radiation pressure on $m_{3}$ due to $m_{1}$ and $\boldsymbol{F}_{\boldsymbol{A}}$ is the Albedo force (solar radiation reflected by $m_{2}$ in space) on $m_{3}$ due to $m_{2}$ (Fig. 1). Let the line joining $m_{1}$ and $m_{2}$ be taken as $X$-axis and $O$ their center of mass as origin. Let the line passing through $O$ and perpendicular to $O X$ and lying in the plane of motion $m_{1}$ and $m_{2}$ be the $Y$ axis. Let us consider a synodic system of co-ordinates $O x y z$ initially coincide with the inertial system $O X Y Z$, rotating with angular velocity $\omega$ about $Z$-axis (the $z$-axis is coincide with $Z$-axis). We wish to find the equations of motion of $m_{3}$ using the terminology of Szebehely [3] in the synodic co-ordinate system and dimensionless variables i.e. the distance between the primaries is unity, the unit of time $t$ is such that the gravitational constant $G=1$ and the sum of the masses of the primaries is unity i.e. $m_{1}+m_{2}=1$.

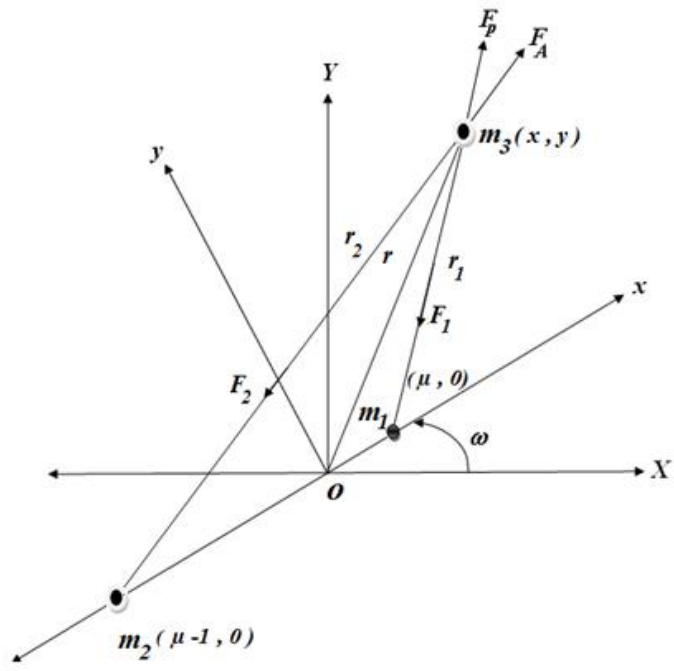

Fig. 1: The Configuration of the R3BP under Albedo effect

The force acting on $m_{3}$ due to $m_{1}$ and $m_{2}$ is $F_{1}\left(1-F_{p} / F_{1}\right)=F_{1}(1$ $-\alpha)$ and $F_{2}\left(1-F_{A} / F_{2}\right)=F_{2}(1-\beta)$ respectively, where $\alpha=F_{p} / F_{1}$ $<<1$ and $\beta=F_{A} / F_{2} \ll 1$. Also, $\alpha$ and $\beta$ can be expressed as:

$$
\alpha=\frac{L_{1}}{2 \pi G m_{1} c \sigma} ; \beta=\frac{L_{2}}{2 \pi G m_{2} c \sigma} \text {; }
$$

where $L_{1}$ is the luminosity of the large primary $m_{1}, L_{2}$ is the luminosity of small primary $m_{2}, G$ is the gravitational constant, $c$ is the speed of velocity and $\sigma$ is mass per unit area.

Now,

$\frac{\beta}{\alpha}=\frac{m_{1}}{m_{2}} \frac{L_{2}}{L_{1}} \Rightarrow \beta=\alpha\left(\frac{1-\mu}{\mu}\right) k$

$k=\frac{L_{2}}{L_{1}}=$ constant

The equations of motion of the infinitesimal mass $m_{3}$ in the synodic coordinate system and dimensionless variables are given by

$$
\begin{aligned}
& \ddot{x}-2 n \dot{y}=\Omega_{x} \\
& \ddot{y}+2 n \dot{x}=\Omega_{y}
\end{aligned}
$$

where

$$
\begin{aligned}
& \Omega=\frac{n^{2}}{2}\left[(1-\mu) r_{1}^{2}+\mu r_{2}^{2}\right]+\frac{(1-\mu)(1-\alpha)}{r_{1}}+\frac{\mu(1-\beta)}{r_{2}}, \\
& \Omega_{x}=n^{2} x-\frac{(1-\mu)(x-\mu)(1-\alpha)}{r_{1}^{3}}-\frac{\mu(x+1-\mu)(1-\beta)}{r_{2}^{3}},
\end{aligned}
$$

$\Omega_{y}=y\left[n^{2}-\frac{(1-\mu)(1-\alpha)}{r_{1}^{3}}-\frac{\mu(1-\beta)}{r_{2}^{3}}\right]$, $n=1$ is the mean motion of the primaries,

$r_{1}^{2}=(x-\mu)^{2}+y^{2}$,

$r_{2}^{2}=(x+1-\mu)^{2}+y^{2}$,

$\mathrm{O}<\mu=\frac{m_{2}}{m_{1}+m_{2}}<\frac{1}{2} \Rightarrow m_{1}=1-\mu ; m_{2}=\mu$.

\section{Libration Points}

At the libration points all the derivatives are zero i.e. $\dot{x}=\mathrm{O}, \dot{y}=\mathrm{O}, \ddot{x}=\mathrm{O}, \ddot{y}=\mathrm{O}, \Omega_{x}=\mathrm{O}$ and $\Omega_{y}=\mathrm{O}$.

Therefore, the libration points are the solutions of the equations $\Omega_{x}=n^{2} x-\frac{(1-\mu)(x-\mu)(1-\alpha)}{r_{1}^{3}}-\frac{\mu(x+1-\mu)(1-\beta)}{r_{2}^{3}}=0$

and

$\Omega_{y}=y\left[n^{2}-\frac{(1-\mu)(1-\alpha)}{r_{1}^{3}}-\frac{\mu(1-\beta)}{r_{2}^{3}}\right]=0$.

\subsection{Non-collinear Libration Points}

The non-collinear libration points are the solution of the Equations $\Omega_{x}=0$ and $\Omega_{y}=0, y \neq 0$ i.e.

$$
\begin{aligned}
& x-\frac{(1-\mu)(x-\mu)(1-\alpha)}{r_{1}^{3}}-\frac{\mu(x+1-\mu)(1-\beta)}{r_{2}^{3}}=0, \\
& 1-\frac{(1-\mu)(1-\alpha)}{r_{1}^{3}}-\frac{\mu(1-\beta)}{r_{2}^{3}}=0,
\end{aligned}
$$

On substituting $\alpha=0$, the solution of Eqns. (6) and (7) is $r_{1}=1, r_{2}$ $=1$. Now we assume that the solution of Eqns. (6) and (7) for $\alpha \neq$ 0 as $r_{1}=1+\xi_{1}, \quad r_{2}=1+\xi_{2}, \xi_{1}, \xi_{2}<<1$.

Substituting these values of $r_{1}$ and $r_{2}$ in the Eqns. (4) and (5), we get

$$
\begin{aligned}
& x=\mu-\frac{1}{2}+\xi_{2}-\xi_{1} \\
& y= \pm \frac{\sqrt{3}}{2}\left[1+\frac{2}{3}\left(\xi_{2}+\xi_{1}\right)\right]
\end{aligned}
$$

Now, substituting the values of $x, y$ from Eqns. (8), $r_{1}=1+\xi_{1}, r_{2}$ $=1+\xi_{2}$ in the Eqns. (6) and (7) and neglecting higher order terms, we obtain

$\xi_{1}=-\frac{1}{3} \alpha, \xi_{2}=-\frac{1}{3} \beta$.

Thus, the coordinates of the non-collinear libration points $L_{4,5}$ are $x=\mu-\frac{1}{2}+\frac{1}{3}(\alpha-\beta)$,

$y= \pm \frac{\sqrt{3}}{2}\left[1+\frac{2}{3}\left\{-\frac{1}{3}(\alpha+\beta)\right\}\right]$

Using the relation (1) i.e. $\beta=\alpha(1-\mu) k / \mu$, we have

$$
x=\mu-\frac{1}{2}+\frac{\alpha}{3}\left[1-\frac{(1-\mu) k}{\mu}\right],
$$

$$
y= \pm \frac{\sqrt{3}}{2}\left[1+\frac{2}{3}\left\{-\frac{\alpha}{3}\left[1+\frac{(1-\mu) k}{\mu}\right]\right\}\right]
$$

Thus, we conclude that there exist two non-collinear libration points $L_{4,5}$ and these points are affected by Albedo effect (Fig. 2(a) (2) \& 2(b)), also these points form scalene triangle with the primaries as $r_{1} \neq r_{2}$. For $\mu=0.1$ and different values of $\alpha$ and $k$ and it is found that the abscissa and ordinate of non-collinear libration points are the decreasing functions of $\alpha$ and $k$ i.e. as $\alpha$ and $k$ increases, $x$ and $y$ decreases.

If $k=0$, the results are in conformity with Bhatnagar \& Chawla [10] and for $\alpha=0$, the classical case of the restricted three body problem is verified (Szebehely [3]). 


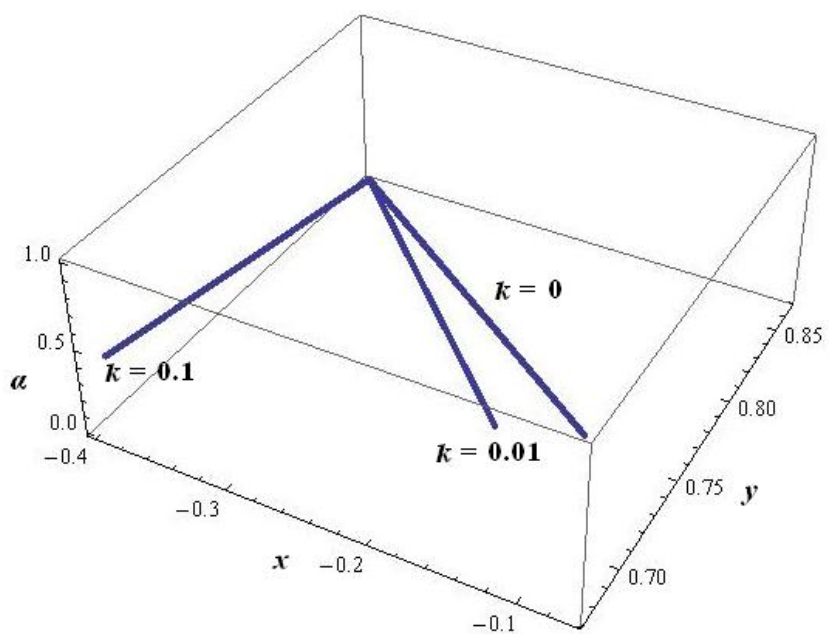

Fig. 2: $L_{4}$ versus $\alpha ; \mu=0.1$

\subsection{Collinear Libration Points}

The collinear libration points are the solution of the Equations $\Omega$ $=0$ and $y=0$ i.e.

$$
f(x)=n^{2} x-\frac{(1-\mu)(x-\mu)(1-\alpha)}{r_{1}^{3}}-\frac{\mu(x+1-\mu)(1-\beta)}{r_{2}^{3}}=0
$$

where $r_{i}=\left|x-x_{i}\right|, i=1,2$, is a seventh degree equation in $x$.

Since $f(x)>0$ in each of the open intervals $(-\infty, \mu-1),(\mu-1, \mu)$ and $(\mu, \infty)$, the function $f(x)$ is strictly increasing in each of them. Also, $f(x) \rightarrow-\infty$ as $x \rightarrow-\infty,(\mu-1)+0$ or $\mu+0$ and $f(x) \rightarrow \infty$ as $x \rightarrow \infty,(\mu-1)-0$ or $\mu-0$. Therefore, there exists only one value of $x$ in each of the open intervals $(-\infty, \mu-1),(\mu-1, \mu)$ and $(\mu$, $\infty)$ such that $f(x)=0$. Further, $f(\mu-2)<0, f(0) \geq 0$ and $f(\mu+1)>0$. Therefore, there are only three real roots lying in each of the intervals $(\mu-2, \mu-1),(\mu-1,0)$ and $(\mu, \mu+1)$. Thus there are only three collinear libration points.

From the Fig. 3(a), this is observed that the first collinear libration point $L_{1}$ always lie at the right of the primary $m_{2}$, the second libration point $L_{2}$ lies between the center of mass of the primaries $O$ and $m_{1}$ and the third libration point $L_{3}$ lies at the right of the primary $m_{1}$. This is also observed that the libration points $L_{1}$ and $L_{3}$ move away from the center of mass as $\mu$ increases while the second libration point $L_{2}$ moves toward the center of mass as $\mu$ increases.

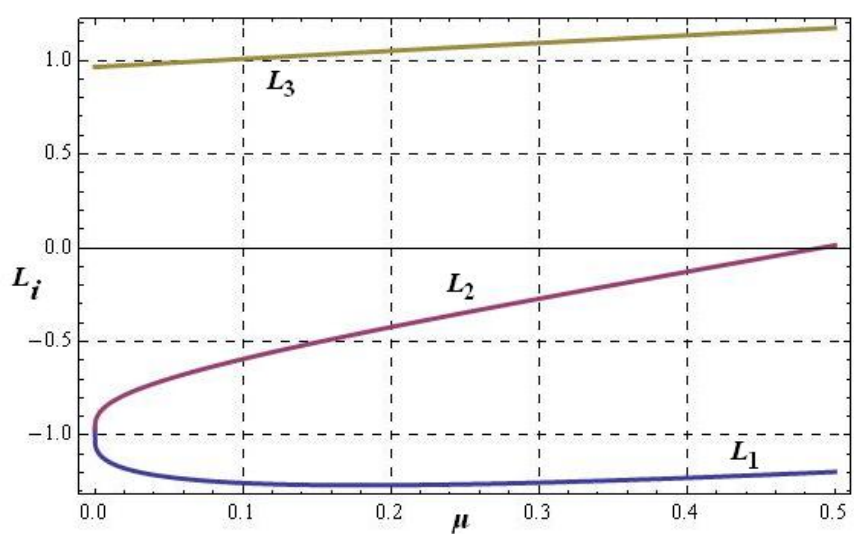

Fig. 3(a): $\mu$ versus $L_{i}(i=1,2,3) ; \alpha=0.1, k=0.05$

The Equation (11) determines the location of the collinear libration points $L_{1}\left(x_{1}, 0\right), L_{2}\left(x_{2}, 0\right)$ and $L_{3}\left(x_{3}, 0\right)$ lie in the intervals $(-\infty, \mu-1),(\mu-1, \mu)$ and $(\mu, \infty)$ respectively, where

$$
\begin{aligned}
& x_{1}=\mu-1-\xi_{1}, \\
& x_{2}=\mu-1+\xi_{2}, \\
& x_{3}=\mu+\xi_{3} .
\end{aligned}
$$

Since the libration point $L_{1}$ lies in the interval $(-\infty, \mu-1)$ i.e. left to the smaller primary, we have $r_{1}=\mu-x_{1}$ and $r_{2}=\mu-1-x_{1}$ which when substituted in Equation (11), gives

$$
n^{2} x+\frac{(1-\mu)(x-\mu)(1-\alpha)}{r_{1}^{3}}+\frac{\mu(x+1-\mu)(1-\beta)}{r_{2}^{3}}=0
$$

Similarly, for $L_{2}\left(x_{2}, 0\right)$ and $L_{3}\left(x_{3}, 0\right)$ the Equation (11) becomes

$$
\begin{aligned}
& n^{2} x+\frac{(1-\mu)(x-\mu)(1-\alpha)}{r_{1}^{3}}-\frac{\mu(x+1-\mu)(1-\beta)}{r_{2}^{3}}=0 \\
& n^{2} x-\frac{(1-\mu)(x-\mu)(1-\alpha)}{r_{1}^{3}}-\frac{\mu(x+1-\mu)(1-\beta)}{r_{2}^{3}}=0
\end{aligned}
$$

For $k=0$, the solutions obtained for the Equations (13), (14) and (15) are the libration points $L_{i}(i=1,2,3)$ in the photogravitational restricted three-body problem studied by Bhatnagar and Chawla in 1979 but if $k \neq 0$ the libration points $L_{i}(i=1,2,3)$ are affected by the Albedo effect and this effect displaced the libration points from its actual position as shown in Fig. 3(b), 3(c) and 3(d).

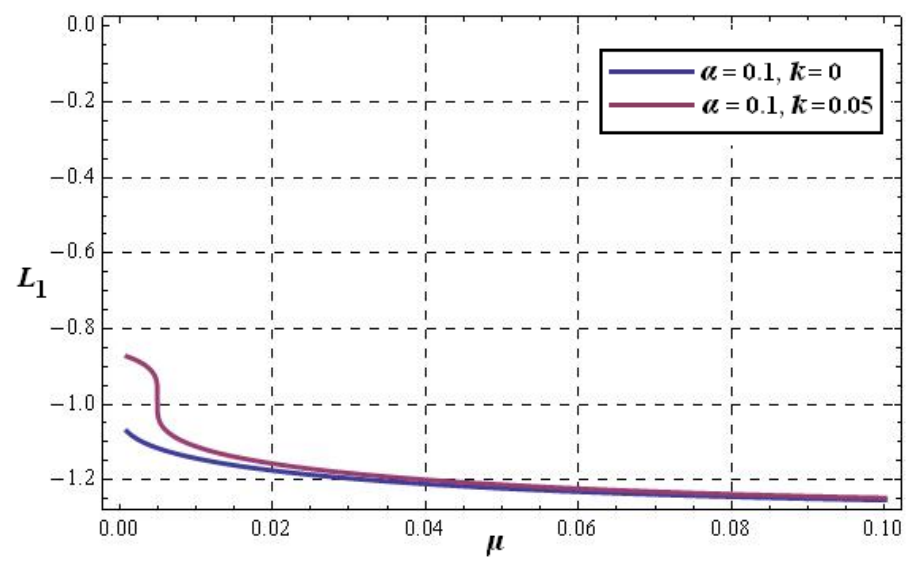

Fig. 3(b): $\mu$ versus $L_{1}$

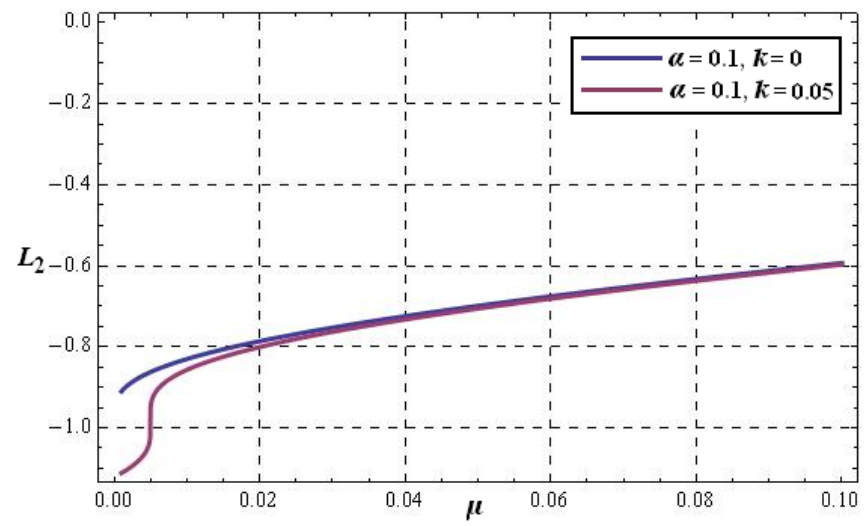

Fig. 3(c): $\mu$ versus $L_{2}$ 


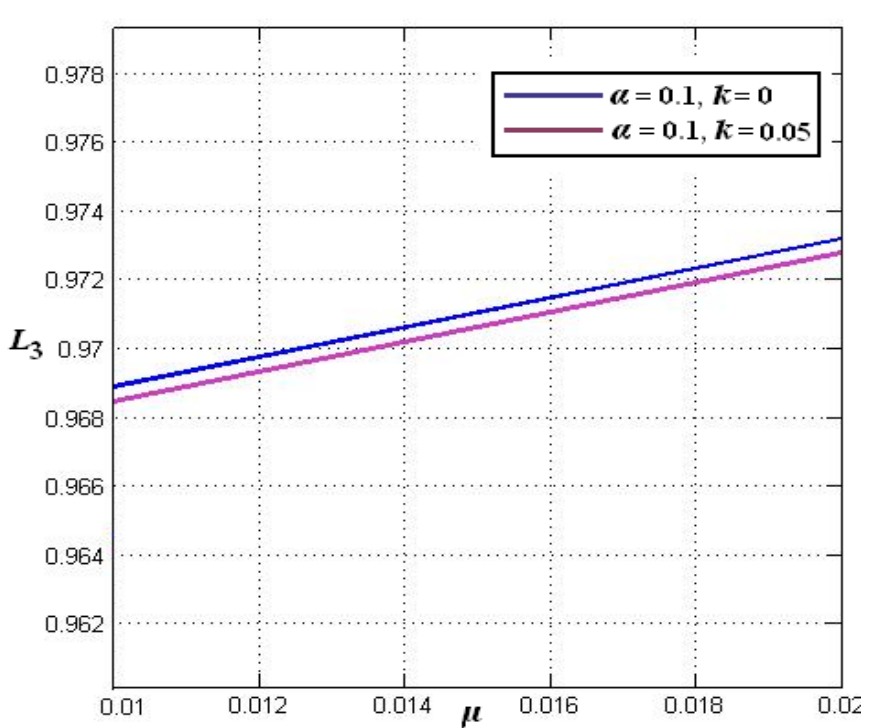

Fig. 3(d): $\mu$ versus $L_{3}$

\section{Stability of Libration Points}

The variational equations are obtained by substituting $x=x_{0}+\xi$ and $y$ $=y_{0}+\eta$ in the equations of motion (2), where $\left(x_{0}, y_{0}\right)$ are the coordinates of $L_{4}$ or $L_{5}$ and $\xi, \eta \ll 1$ i.e.

$$
\begin{aligned}
& \ddot{\xi}-2 n \dot{\eta}=\xi \stackrel{o}{\Omega}_{x x}+\eta \stackrel{o}{\Omega_{x y}}, \\
& \ddot{\eta}+2 n \dot{\xi}=\xi \stackrel{o}{\Omega}_{x y}+\eta \stackrel{o}{\Omega}_{y y} .
\end{aligned}
$$

Here we have taken only linear terms in $\xi$ and $\eta$. The subscript in $\Omega$ indicates the second partial derivative of $\Omega$ and superscript $o$ indicates that the derivative is to be evaluated at the libration point $\left(x_{0}, y_{0}\right)$. The characteristic equation corresponding to Eqn. (16) is

$$
\lambda^{4}+\left(4 n^{2}-\stackrel{o}{\Omega}_{x x}-\stackrel{o}{\Omega}_{y y}\right) \lambda^{2}+\stackrel{o}{\Omega}_{x x} \stackrel{o}{\Omega}_{y y}-\left(\stackrel{o}{\Omega}_{x y}\right)^{2}=0
$$

where

$$
\begin{aligned}
& \stackrel{o}{\Omega}_{x x}=\frac{3}{4}\left[1-\frac{2}{3}(1-3 \mu) \alpha+\frac{2}{3}(2-3 \mu) \beta\right], \\
& \stackrel{o}{\Omega}_{x y}=\frac{3 \sqrt{3}}{2}\left[\mu-\frac{1}{2}+\frac{1}{9}(1+\mu) \alpha-\frac{1}{9}(2-\mu) \beta\right], \\
& \stackrel{o}{\Omega}_{y y}=\frac{9}{4}+\frac{1}{2}(1-3 \mu) \alpha-\frac{1}{2}(2-3 \mu) \beta .
\end{aligned}
$$

\subsection{Stability of Non-collinear Libration points}

Let $\lambda^{2}=\Pi$, therefore Equation (17) becomes

$$
\Pi^{2}+q_{1} \Pi+q_{2}=\mathrm{O}
$$

which is a quadratic equation in $\Pi$ and its roots are given by

$\Pi_{1,2}=\frac{1}{2}\left(-q_{1} \pm \sqrt{D}\right)$

where

$q_{1}=4 n^{2}-\stackrel{o}{\Omega}_{x x}-\stackrel{o}{\Omega}_{y y} ; q_{2}=\stackrel{o}{\Omega}_{x x} \stackrel{o}{\Omega}_{y y}-\left(\stackrel{o}{\Omega}_{x y}\right)^{2} ; D=q_{1}^{2}-4 q_{2}$.

The motion near the Libration point $\left(x_{0}, y_{0}\right)$ is said to be bounded if all the four roots of Equation (17) are pure imaginary which is possible if and only if the discriminant of Equation (18) is zero i.e.

$$
1-27 \mu+27 \mu^{2}-6(1-\mu)[\mu+(1-\mu) k] \alpha=0
$$

The Equation (20) is quadratic in $\mu$, on solving it we have

$$
\mu_{1,2}=\frac{9 \pm \sqrt{69}}{18} \pm \frac{(2 \sqrt{69} \mp 207 k+25 \sqrt{69} k) \alpha}{1863}+\mathrm{O}\left(\alpha^{2}\right)
$$

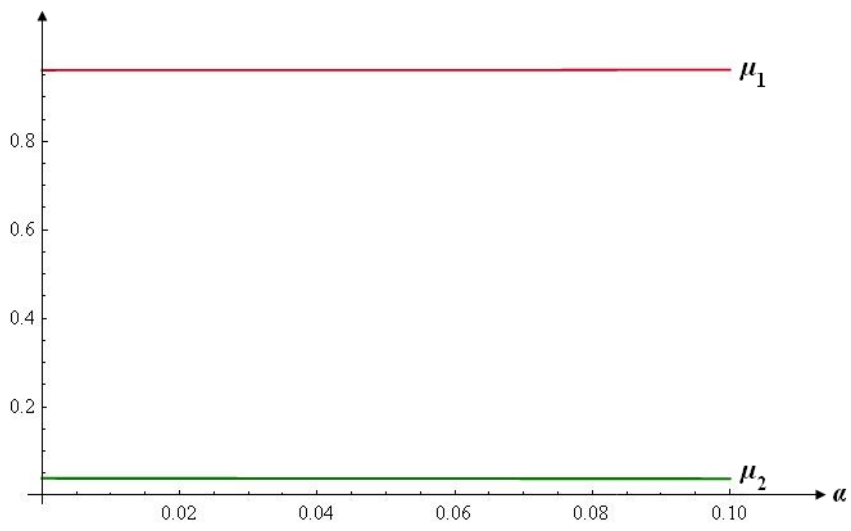

Fig. 4: $\mu$ versus $\alpha ; k=0.01$

From the Fig. (4), $\mu_{1}>1 / 2$ and $\mu_{2}<1 / 2$ for all values of $\alpha$. Thus the critical value of mass parameter $\mu_{\mathrm{c}}$ for which the non-collinear libration points $L_{4,5}$ are stable is

$$
\mu_{c}=\frac{9-\sqrt{69}}{18}-\frac{(2 \sqrt{69}+207 k+25 \sqrt{69} k) \alpha}{1863}<\frac{1}{2} \text {. }
$$

On simplifying we have

$\mu_{c}=\mu_{\mathrm{o}}-(0.00891747+0.222579 k) \alpha$,

where $\mu_{o}=0.038520896504551 \ldots$ is the critical value of mass parameter for classical case. If $\alpha=0$ then $\mu=\mu_{\mathrm{o}}$ is the solution of the Equation (20) (Szebehely [3]) and for $k=0, \mu=\mu_{o}-$ $0.00891747 \alpha$ is the solution of the Equation (20) (Bhatnagar and Chawla [10]).

Thus, the non-collinear libration points $L_{4,5}$ are stable for the critical mass parameter $\mu \leq \mu_{\mathrm{c}}$, where $\mu_{\mathrm{c}}$ is defined in Equation (22).

\subsection{Stability of Collinear Libration points}

First we consider the point lying in the interval $(\mu-2, \mu-1)$. For this point, $r_{2}<1, r_{1}>1$ and

$$
\begin{aligned}
& \stackrel{o}{\Omega}_{x x}=n^{2}+\frac{2(1-\mu)(1-\alpha)}{r_{1}^{3}}+\frac{2 \mu(1-\beta)}{r_{2}^{3}}>0, \stackrel{o}{\Omega}_{x y}=0, \\
& \stackrel{\circ}{\Omega_{y y}}=\mu\left(\frac{1}{r_{2}}-\frac{1}{r_{1}}\right)\left(r_{2}-\frac{1}{r_{2}^{2}}\right)+\frac{\mu}{r_{2}}\left(\frac{1}{r_{2}}-\frac{1}{r_{1}}\right) \beta<0 .
\end{aligned}
$$

Similarly, for the points lying in the interval $(\mu-1,0)$ and $(\mu, \mu+$ 1), $\stackrel{\circ}{\Omega}_{x x}>0, \stackrel{\circ}{\Omega}_{x y}=0, \stackrel{\circ}{\Omega}_{y y}<0$. Since the discriminant of Equation (18) is positive and the four roots of the characteristic equation (17) an be written as $\lambda_{1,2}= \pm s$ and $\lambda_{3,4}= \pm i t$ ( $s$ and $t$ are real). Hence the motion around the collinear libration points is unbounded and consequently the collinear libration points are unstable.

\section{Conclusion}

In the present paper, the existence and stability of libration points in circular restricted three-body problem has been studied under Albedo effect. The equations of motion in case of Albedo effect are derived, Eqn. (2). For $\beta=0$, the problem reduces to photogravitational restricted three-body problem. It is found that there exist five libration points, three collinear and two non-collinear. The first collinear libration point $L_{1}$ lie at the right of the primary $m_{2}$, the second libration point $L_{2}$ lies between the center of mass of the primaries $O$ and $m_{1}$ and the third libration point $L_{3}$ lies at the right 
of the primary $m_{1}$. The libration points $L_{i}(i=1,2,3)$ are affected by the Albedo effect and this effect displaced the libration points from its actual position as shown in Fig. 3(b), 3(c) and 3(d). Also, there exist two non-collinear libration points $L_{4,5}$ and these points are also affected by Albedo (Fig. 2(a) \& 2(b)), these points form scalene triangle with the primaries as $r_{1} \neq r_{2}$. For $k=0$, the results are agreed with Bhatnagar and Chawla [10] and if we take $\alpha=0$ and $k=0$, the classical case of the restricted three body problem is verified (Szebehely [3]). The non-collinear libration points are stable for a critical value of mass parameter $\mu \leq \mu_{c}$, where $\mu_{\mathrm{c}}=\mu_{o}$ - $(0.00891747+0.222579 k) \alpha$ but collinear libration points are still unstable.

\section{References}

[1] Danby, J.M.A.: Stability of the triangular points in the elliptic restricted problem of three bodies. The Astronomical Journal, 69 (2), 165 - 172 (1964).https://doi.org/10.1086/109254.

[2] Szebehely, V.: Theory of orbits, The Restricted Problem of three bodies. Academic Press, New York and London (1967).

[3] Harris, M., Lyle, R.: Spacecraft Radiation Torque. NASA Space Vehicle Design Criteria (Guidance and Control) NASA SP-8027, 137, October 1969.

[4] Chernikov, Y.A.: The photogravitational restricted three body problem. Soviet Astronomy-AJ, 14, 176-181 (1970).

[5] Vidyakin, V.V.: Stability of one particular solution for the motion of three homogeneous spheroids.Soviet Astronomy, 18, 116 (1974).

[6] Sharma, R.K.: Perturbations of Lagrangian points in the restricted three-body problem. Indian Journal of Pure and Applied Mathematics, 6, 1099-1102 (1975)

[7] Subbarao, P.V., Sharma, R.K.: A note on the Stability of the triangular points of equilibrium in the restricted three body problem. Astronomy and Astrophysics, 43, 381-383 (1975).

[8] Sharma, R.K., Subbarao P.V.: Stationary solutions and their characteristic exponents in the restricted three-body problem when the more massive primary is an oblate spheroid. Celestial Mechanics $\begin{array}{llll}\text { and } & \text { Dynamical } & \text { Astronomy, 137 }\end{array}$ (1976).https://doi.org/10.1007/BF01232721.

[9] Choudhary R.K.: Libration points in the generalized elliptic restricted three body problem. Celestial Mechanics, 16, $411-419$ (1977).https://doi.org/10.1007/BF01229285.

[10] Bhatnagar K. B., Hallan P. P.: Effect of perturbed potentials on the stability of libration points in the restricted problem, Celestial Me-

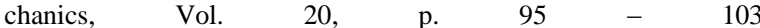
(1979).https://doi.org/10.1007/BF01230231.

[11] Bhatnagar, K.B., Chawla, J.M.: A study of Lagrangian points in the photogravitational restricted three-body problem. Indian J. Pure Appl. Math. 10(11), 1443-1451 (1979).

[12] Schuerman, D.W.: The restricted three body problem including radiation pressure. The Astrophysical Journal, 238, 337-342 (1980).https://doi.org/10.1086/157989.

[13] Anselmo, L., Farinella, P., Milani, A., Nobili, A. M.: Effects of the Earth reflected sunlight on the orbit of the LAGEOs satellite. Astronomy and Astrophysics. 117 (1), 1 - 3 (1983).

[14] Cid, R., Ferrer, S., Caballero, J.A.: Asymptotic solutions of the restricted problem near equilateral Lagrangian points. Celestial Mechanics and Dynamical Astronomy, 35, 189-200 (1985).https://doi.org/10.1007/BF01227668.

[15] Kunitsyn, A.L., Tureshbaev, A.T.: On the collinear libration points in the photogravitational three-body problem. Celest. Mech. 35(2), 105-112 (1985). https://doi.org/10.1007/BF01227664.

[16] Simmons, J.F.L., McDonald, A.J.C., Brown, J.C.: The restricted 3 body problem with radiation pressure. Celestial Mechanics and Dynamical Astronomy, 35, 145-187 (1985).https://doi.org/10.1007/BF01227667.

[17] Sharma, R.K.: The linear stability of libration points of the photogravitational restricted three-body problem when the smaller primary is an oblate spheroid. Astrophys. Space Sci. 135(2), 271-281 (1987).https://doi.org/10.1007/BF00641562.

[18] Lukyanov, L.G.: On the family of the libration points in the restricted photogravitational three-body problem. Astron. Zh. 65 , 422-432 (1988).

[19] El-Shaboury, S.M.: Equilibrium solutions of the restricted problem of $2+2$ axisymmetric rigid bodies. Celestial Mechanics and Dynamical Astronomy, 50, 199-208 (1991).https://doi.org/10.1007/BF00048764.
[20] Lang, K. R.: Astrophysical Data: Planets and Stars. SpringerVerlag, New York (1992).https://doi.org/10.1007/978-1-46840640-5.

[21] Xuetang, Z.H.E.N.G., Lizhong, Y.U.: Photogravitationally restricted three-body problem and coplanar libration point. Chinese Phys. Lett., 10 (1), 61 - 64 (1993).https://doi.org/10.1088/0256307X/10/1/017.

[22] Bhatnagar, K.B., Gupta, U., Bharadwaj, R.: Effect of perturbed potentials on the non-linear stability of Libration point $\mathrm{L}_{4}$ in the restricted problem. Celestial Mechanics and Dynamical Astronomy, 59, 45-374 (1994).https://doi.org/10.1007/BF00692102.

[23] Murray, C.D.: Dynamical effects of drag in the circular restricted three body problems: 1.Location and stability of the Lagrangian equilibrium points. Icarus 112, 465-184 (1994).https://doi.org/10.1006/icar.1994.1198.

[24] Selaru, D., Cucu-Dumitrescu, C.: Infinitesimal orbits around Lagrange points in the elliptic, restricted three-body problem. Celestial Mechanics and Dynamical Astronomy, 61 (4), 333 - 346 (1995).https://doi.org/10.1007/BF00049514.

[25] Markellos, V.V., Papadakis, K.E., Perdios, E.A.: Non-linear stability zones around triangular equilibria in the plane circular restricted three-body problem with oblateness. Astrophysics and Space Science, 245, 157-164 (1996). https://doi.org/10.1007/BF00637811.

[26] Subbarao, P.V., Sharma, R.K.: Effect of oblateness on the nonlinear stability of $\mathrm{L}_{4}$ in the restricted three-body problem. Celestial Mechanics and Dynamical Astronomy, 65, 291-312 (1997).

[27] Khanna, M., Bhatnagar, K.B.: Existence and stability of libration points in restricted three body problem when the smaller primary is a triaxial rigid body. Indian Journal of Pure and Applied Mathematics, 29 (10), 1011-1023 (1998).

[28] Nuss, J. S.: The use of solar sail in the circular restricted problem of three bodies. Master's Thesis, Purdue University (1998).

[29] Khanna, M., Bhatnagar, K.B.: Existence and stability of Libration points in the restricted three body problem when the smaller primary is a triaxial rigid body and the bigger one an oblate spheroid. Indian Journal of Pure and Applied Mathematics, 30 (7), 721-733 (1999).

[30] Murray, C.D., Dermott, S.F.: Solar System Dynamics. Cambridge University Press, Cambridge (1999).

[31] McInnes, A. I. S.: Strategies for solar sail mission design in the circular restricted three-body problem. Master's Thesis, Purdue University (2000)

[32] Grün, E., Gustafson, B.A.S., Dermott, S., Fechtig, H.: Interplanetary Dust. Springer, Berlin (2001).https://doi.org/10.1007/978-3-64256428-4.

[33] Roberts, G.E.: Linear Stability of the Elliptic Lagrangian Triangle Solutions in the three-body problem. Journal of Differential Equations, $\quad 182 \quad(1), \quad 191$ (2002).https://doi.org/10.1006/jdeq.2001.4089.

[34] Oberti, P.,Vienne, A.: An upgraded theory for Helene, Telesto and Calypso. Astronomy and Astrophysics, 397, 353-359 (2003).https://doi.org/10.1051/0004-6361:20021518.

[35] Bhanderi, D. D. V., Bak, T.: Modeling Earth Albedo Satellites in Earth orbit. AIAA Guidance, Navigation and Control Proceedings, San Franciso, California (2005).https://doi.org/10.2514/6.20056465.

[36] Perdiou, A.E., Markellos, V.V., Douskos, C.N.: The Hill problem with oblate secondary: Numerical Exploration. Earth, Moon and Planets, 97, 127-145 (2005).https://doi.org/10.1007/s11038-0069065-y.

[37] Pontus, A.: Attitude estimation from magnometer and earth-albedocorrected coarse Sun sensor measurements. Journal of ActaAstonautica, 56 (1-2) (2005).

[38] Sosnytskyi, S.P.: On the Lagrange stability of motion in the threebody problem. Ukrainian Mathematical Journal, 57 (8), 1341-1349 (2005).https://doi.org/10.1007/s11253-005-0266-8.

[39] Kushvah, B.S., Sharma, J.P., Ishwar, B.: Nonlinear Stability in the Generalized Photogravitational Restricted Three Body Problem with Poynting-Robertson Drag. Astrophysics and Space Science, 312, 279-293 (2007). https://doi.org/10.1007/s10509-007-9688-0.

[40] Ammar, M.K.: The effect of solar radiation pressure on the Lagrangian points in the elliptic restricted three-body problem. Astrophysics and Space Science, 313 (4), 393 - 408 (2008). https://doi.org/10.1007/s10509-007-9709-z.

[41] Rocco, E. M.: Evaluation of the Terrestial Albedo Applied to Some Scientific Missions. Space Science Review, 151 (1-3), 135-147 (2009).

[42] Douskos, C.N.: Collinear equilibrium points of Hill's problem with radiation and oblateness and their fractal basins of attraction. As- 
trophysics and Space Science, 326, 263-271 (2010).https://doi.org/10.1007/s10509-009-0213-5.

[43] Singh, Jagdish, Leke, O: Stability of the photogravitational restricted three body problem with variable masses. Astrophysics and Space Science, 326, 305-314 (2010).https://doi.org/10.1007/s10509-009-0253-x.

[44] Celletti, A., Stefanelli, L., Lega, E., Froeschlé, C.: Some Results on the Global Dynamics of the Regularized Restricted Three-Body Problem with Dissipation. Celestial Mechanics and Dynamical Astronomy, 109, 265-284 (2011).https://doi.org/10.1007/s10569-0109326-y.

[45] MacDonald, M., McInnes, C.: Solar sail science mission applications and advancement. Advances in Space Research, 48, 17021716 (2011).https://doi.org/10.1016/j.asr.2011.03.018.

[46] Ershkov, Sergey V.: The Yarkovsky effect in generalized photogravitational 3-body problem. Planetary and Space Science, 70 (1), 221-223 (2012).https://doi.org/10.1016/j.pss.2012.09.002.

[47] John, A. Arredondo, Jianguang, Guo, Cristina, Stoica, Claudia, Tamayo: On the restricted three body problem with oblate primaries. Astrophysics and Space Science, 341, 315-322 (2012).https://doi.org/10.1007/s10509-012-1085-7.

[48] Idrisi, M. Javed, Taqvi, Z.A.: Restricted three-body problem when one of the primaries is an ellipsoid. Astrophysics and Space Science, 348, 41-56 (2013).https://doi.org/10.1007/s10509-0131534-y.

[49] Idrisi, M. Javed: Existence and stability of the libration points in CR3BP when the smaller primary is an oblate spheroid. Astrophysics and Space Science, 354, 311-325 (2014).https://doi.org/10.1007/s10509-014-2031-7.

[50] Katour, D.A., Abd El-Salam, F.A., Shaker, M.O.: Relativistic restricted three body problem with oblateness and photo-gravitational corrections to triangular equilibrium points. Astrophysics and Space Science, 351, 143 - 149 (2014).https://doi.org/10.1007/s10509-0141826-X.

[51] Idrisi, M. Javed, Amjad, Muhammad: Effect of elliptic angle $\varphi$ on the existence and stability of libration points in restricted threebody problem in earth-moon system considering earth as an ellipsoid. International Journal of Advanced Astronomy, 3 (2), 87-96 (2015).https://doi.org/10.14419/ijaa.v3i2.5313.

[52] Jain, Mamta, Aggarwal, Rajiv: Restricted three body problem with Stokes Drag effect. International Journal of Astronomy and Astrophysics, 5, 95-105 (2015).https://doi.org/10.4236/ijaa.2015.52013.

[53] Jain, Mamta, Aggarwal, Rajiv: A study of non-collinear libration points in restricted three body problem with stokes drag effect when smaller primary is an oblate spheroid. Astrophysics and Space Science, 358: 51 (2015).https://doi.org/10.1007/s10509-015-24576.

[54] Lhotka, C., Celletti, A.: The effect of Poynting Robertson drag on the triangular Lagrangian points. Astrophysics and Space Science, 250, 249-261 (2015).https://doi.org/10.1016/j.icarus.2014.11.039.

[55] Mishra, Vivek Kumar, Ishwar, Bhola: Normalisation of Hamiltonian in photogravitational elliptic restricted three body problem with Poynting-Robertson drag. International Journal of Advanced Astroomy, 3, 42-45 (2015). https://doi.org/10.14419/ijaa.v3i1.4534.

[56] Pal, Ashok Kumar, Kushvah, Badam Singh: Geometry of halo and lissajous orbits in the circular restricted three body problem with drag forces.MNRAS446, 959-972 (2015).

[57] Singh, J., Amuda, T.O.: Out-of-plane equilibrium points in the photogravitational CR3BP with oblateness and P-R drag. Journal of Astrophysics and Astronomy, 36, 291-305 (2015).https://doi.org/10.1007/s12036-015-9336-y.

[58] Idrisi, M.J..: A general solution to mon-collinear equilibria in terms of largest root $(\kappa)$ of confocal oblate spheroid. International Journal of Advanced Astronomy, 4(1), 1 - 4 (2016).https://doi.org/10.14419/ijaa.v4i1.5587

[59] Idrisi, M.J., Kumari, Shalini: Non-collinear libration points in CR3BP when less massive primary is an heterogeneous oblate body with N-layers. International Journal of Advanced Astronomy, 4(1), 39 - 42 (2016).https://doi.org/10.14419/ijaa.v4i1.5928.

[60] Idrisi, M.J., Jain, Mamta: Non-collinear libration points in CR3BP when less massive primary is an heterogeneous oblate body with $\mathrm{N}$ layers. International Journal of Advanced Astronomy, 4(1), 61 - 67 (2016).https://doi.org/10.14419/ijaa.v4i1.6140. 